\title{
Relaxing symmetry rules for nonlinear optical interactions via strong light-matter coupling
}

Mandeep Khatoniar

City College \& Grad Center of CUNY

Rezlind Bushati

City College \& Grad Center of CUNY

Ahmed Mekkawy

ASRC Photonics Initiative and EE Dept. CCNY

Florian Dirnberger

City College of New York

\section{Andrea Alu}

CUNY Advanced Science Research Center https://orcid.org/0000-0002-4297-5274

Vinod Menon ( $\square$ vmenon@ccny.cuny.edu )

City College of New York https://orcid.org/0000-0002-9725-6445

\section{Research Article}

Keywords: Transition Metal Dichalcogenides (TMDCs), optoelectronic devices, Second Harmonic Generation (SHG)

Posted Date: May 20th, 2021

DOI: https://doi.org/10.21203/rs.3.rs-512787/v1

License: (c) (i) This work is licensed under a Creative Commons Attribution 4.0 International License.

Read Full License 


\title{
Relaxing Symmetry Rules for Non-Linear Optical Interactions via Strong Light-Matter Coupling
}

\author{
Mandeep Khatoniar ${ }^{1,2, \ddagger}$, Rezlind Bushati $^{1,2, \ddagger}$, Ahmed Mekkawy ${ }^{3,4}$, Florian Dirnberger $^{2}$, \\ Andrea $\mathrm{Alu}^{1,3,4}$,Vinod Menon ${ }^{1,2}$ \\ $\ddagger$ These authors contributed equally to this work
}

${ }^{1}$ Department of Physics, The Graduate Center, City University of New York, New York 10016, United States

2 Department of Physics, City College of New York, City University of New York, New York 10031, United States

${ }^{3}$ Photonics Initiative, Advanced Science Research Center, City University of New York, New York 10031, United States

${ }^{4}$ Department of Electrical Engineering, City College of New York, City University of New York, New York 10031, United States Corresponding Author: vmenon@ccny.cuny.edu.

\begin{abstract}
Transition Metal Dichalcogenides (TMDCs) have been in the limelight for the past decade as a candidate for several optoelectronic devices, and as a versatile test bed for various fundamental light-matter interaction phenomena thanks to their exceptional linear optical properties arising from their large binding energy, strong spin-orbit coupling and valley physics in the monolayer (ML) limit. They also boast strong non-linear properties fortified by excitonic responses in these systems. However, the strong second order non-linear responses are mostly restricted to the ML limit owing to crystal symmetry requirements, posing several limitations in terms of smaller interaction length and lower damage threshold. Here we demonstrate a selfhybridized exciton-polariton system in bulk $\mathrm{WSe}_{2}$ that allows us to relax the crystal symmetry rules that govern second order non-linearities. The demonstrated polariton system shows intense Second Harmonic Generation (SHG) when the fundamental wavelength is resonant with the lower polariton, with an efficiency comparable to the one from a ML $\mathrm{WS}_{2}$ when excited at the same fundamental wavelength and intensity. We model this phenomenon by considering a system with alternating second-order susceptibilities under an asymmetric electric field profile determined by the polariton mode. Helicity resolved polarization experiments show very similar non-linear response as the one from a ML where the helicity of the SHG flips with respect to the fundamental harmonic. The polaritonic system offers a platform to leverage robust second order non-linear response from centrosymmetric systems, while at the same time allowing access to third-order non-linearity inherent in strongly coupled systems.
\end{abstract}

\section{Introduction}

Transition Metal Dichalcogenides (TMDCs) are Van der Waals(vdW) materials that exhibit a vista of much sought after optical and electronic properties. They have been studied extensively in the monolayer (ML) limit due to their direct bandgap, large 
exciton binding energy, valley properties, and oscillator strength [1-4]. Due to these properties, strong coupling between cavity photons in microcavities and excitons in ML TMDCs can been demonstrated at room temperature [5]. In addtion in such a strongly coupled system, the polaritons retain the valley excitonic properties of the host material [6-8]. More recently, there has been interest in polaritons formed in bulk TMDCs due to their high refractive index, which enables Fabry-Perot modes to be sustained in TMDC slabs which strongly couples with the exciton modes of the bulk TMDC [9-11]. This coupling results in a self-hybridized system where the bulk TMDC itself provides both the photonic and excitonic components required for strong coupling, without the need for an external cavity.

In addition to their exceptional linear optical properties, there have also been numerous reports on their nonlinear optical response. Second harmonic generation (SHG) can be observed in TMDCs owing to the lack of inversion symmetry in the ML and odd layer limit [12-15].More recently, SHG in TMDCs has emerged as a powerful spectroscopic tool to characterize the layer number as well as the crystal orientation, the latter being an important parameter for achieving precise twist angle in vdW heterostructures [16-19]. In addition to intense SHG, other second order susceptibility $\chi^{(2)}$ mediated processes like sum frequency generation at continuous wave pump,optical parametric amplification and signatures of spontaneous parametric down-conversion have also been realized in these systems [20-22]. These nonlinear optical responses can be further enhanced by engineering lightmatter interactions with nanophotonic tools. Since 2D TMDCs show high values of nonlinear susceptibility, (e.g.,two-photon absorption in $\mathrm{MoS}_{2}$ is 4 orders of magnitude larger than III-V group semiconductors such as GaAs) they represent an ideal platform for nonlinear optical applications [23]. Despite this advantage, 2D TMDCs suffer from the limitation that second-order nonlinear response is limited to the ML/odd few layer limit, which proves to be a challenge for device integration. Several attempts have been put forward to increase the interaction time via auxiliary systems where a ML or few-layer TMDCs couple to plasmonic or photonic modes in passive [24-26] or active [27] nanostructures.

The restoration of inversion symmetry in bulk TMDC crystals with naturally occurring $2 \mathrm{H}$ stacking, prohibits second order non-linearity under the electric dipole approximation, consequently creating a limitation in terms of the available interaction length. In this work, we achieve strong nonlinear $\chi^{(2)}$ response, despite the presence of inversion symmetry, in self-hybridized exciton polaritons formed via strong coupling of Fabry-Perot modes sustained by a bulk ( $140 \mathrm{~nm}$ ) WSe $\mathrm{W}_{2}$ and the exciton resonances of each layer. The existence of these modes creates a non-zero phase difference between the SHG signal generated in each layer, owing to the asymmetry of the electric field of the fundamental laser at the polariton mode, which when added coherently, does not cancel out in the far field. We compare the polarization response of this polariton system and find they exhibit similar behavior as their ML counterparts. A comparison between a ML TMDC system and these self hybridized polariton systems show that the latter is more efficient at lower powers than the former. The strong coupling assisted approach presented here allows to simultaneously use material systems where crystal symmetry dependent selection rules can be relaxed to recover latent non-linearity whilst leveraging the additional photonic control knobs and the inherent non-linearity of polariton fluids. [28]. 


\section{Results}

Figure 1a shows the schematic of the structure used in this work, which consists of a bulk $\mathrm{WSe}_{2}$ flake dry transferred on top of a $7.5 \mu \mathrm{m}^{2}$ square box that was etched on a 10.5 period Distributed Bragg Reflector(DBR) consisting of alternating TiO2/SiO2 layers. The thickness of the bulk TMDC and the depth of the square hole were optimized using transfer matrix calculations and coupled mode theory such that self-hybridized exciton polaritons are only formed within the square structure, and outside the etched box there are no polaritons are supported. This step ensures homogeneity of the refractive index surrounding region in which polaritons are formed. Moreover a single flake can host both the regions of polariton formation as well as a reference region with no strong coupling. See Methods for details. Figure 1b shows the white light reflection taken at the location of the square box indicating the presence of two new eigenmodes, namely the upper and the lower polariton branches. We fit a coupled oscillator model to obtain a Rabi splitting of $70 \mathrm{meV}$ and a negative cavity mode detuning of $77.5 \mathrm{meV}$. Transfer matrix simulation of white light reflection spectrum taken inside and outside the square region are shown in Supplementary Figure 1. Figure 2a shows the SHG signal (in blue) when the lower polariton is excited with pulsed Ti:Sapphire laser tuned at a wavelength resonant to the minima of the lower polariton branch ( $a t \mathrm{k}_{\|}=0$ ). As a comparison the SHG signal from the region where no polaritons are formed (outside the square) is also shown in Fig. 2a (in red). A sharp signal at half the wavelength of the fundamental is observed in the case of resonant excitation of the polariton branch. The output intensity versus input power dependence in logarithmic scale is plotted in Supplementary Figure 2a., shows a slope of 1.83, which is indicative of SHG . We detect no discernible signal from the region outside the square box, and estimate a lower bound for the enhancement factor based on the detector noise levels. Inside the box the SHG signal is enhanced at least by three orders of magnitude. Figure $2 \mathrm{~b}$ compares the SHG intensity as a function of detuning of the fundamental wavelength from the one at $\mathrm{k}_{\|}=0$. The laser has a linewidth of approximately $5 \mathrm{~nm}$ and the polariton linewidth is $20 \mathrm{~nm}$. We find that the SHG signal is reduced by $80 \%$ for a laser-cavity detuning of $15 \mathrm{~nm}$.

To further characterize the nature of the SHG in the polariton system we resort to polarization dependent studies. We measure the linear polarization response of the SHG by rotating the plane of input linear polarization of the fundamental while keeping the bulk TMDC and an output analyzer stationary. Under this measurement configuration the $\mathrm{D}_{3}$ h symmetry structure should give a four-fold symmetry [29]. See Supplementary Note 3 for details. The inset of Figure 3a. shows the fourfold symmetry as a function of the input polarization of the fundamental excitation. This gives distinctive evidence that the SHG originates from a crystal with a $\mathrm{D}_{3}$ h symmetry group and it can be attributed to the residual phase that the SHG and the fundamental pick up due to multiple reflections within the structure, thereby producing a non-zero SHG in the far field. After confirming the crystal symmetry, we excite the sample with a circularly polarized fundamental. As seen in ML TMDCs, the polariton system also produces a circularly polarized SHG signal. Figure 3a shows that the measured SHG signal when excited with $\sigma^{+}$ fundamental. The helicity of the SHG signal is flipped from the one of the fundamental thereby confirming the $\mathrm{D}_{3} \mathrm{~h}$ symmetry.

We define degree of circular polarization as $\rho=\left|\frac{I^{\sigma^{+}}-I^{\sigma^{-}}}{I^{\sigma^{+}}+I^{\sigma^{-}}}\right|$, where $I^{\sigma^{+}}$is the intensity of right-handed circularly polarized light and $I^{\sigma^{-}}$is the intensity of left-handed circularly polarized light when analyzed through a quarter wave plate and linear polarizer at the output. After normalizing with the degree of circular polarization of the laser,we obtain a value of $\rho=0.87$ for the case of bulk $\mathrm{WSe}_{2}$ polaritons, which is comparable with the reported value $\rho=0.94$ obtained for SHG from a ML $\mathrm{WS}_{2}$ coupled to a 
passive photonic structure [25].

Next, we compare the efficiency of SHG from polaritons with a ML $\mathrm{WS}_{2}$ that was transferred on 300nm thermal oxide on Silicon. $\mathrm{WS}_{2}$ was chosen as an archetypal ML as it has one of the largest value of second order permitivity.The power dependent measurements in Figure 3b. show the ratio between the SHG signal from the $\mathrm{WSe}_{2}$ self-hybridized polariton sample and a reference $\mathrm{WS}_{2} \mathrm{ML}$ which is pumped at the same wavelength. The plot can be divided into three distinct regimes of power. At low pump powers (region I), it can be seen that the efficiency of SHG from the polariton system is higher than the one from a non center-symmetric ML $\mathrm{WS}_{2}$ sample. At intermediate powers (region II) of $\approx 1 \mathrm{~mW}$ there is a drop in the ratio of intensities of SHG generation in the polariton systems and the $\mathrm{ML} \mathrm{WS}_{2}$. This is the region where the Kerr like polariton non-linearity via phase space filling of the excitons kicks in. At higher pump powers (region III), as a consequence of phase space filling the lower polaritons branch shifts towards higher energies, thereby making the pump laser detuned from the lower polariton resonance. This results in overall reduction in efficiency as shown in Figure 3b. This effect results in a deviation from the typical slope of 2 for the SHG signal from the polariton system. ( Supplementary Figure 2a and Supplementary note 4.) Individual plots for SHG intensity comparison for the self-hybridized polaritons and $\mathrm{ML} \mathrm{WS}_{2}$ as a function of pump power are shown in Supplementary Figure 6. Such phase space filling effects have been extensively studied in various ML TMDC systems with interest in the generation of polariton blockade.[30-34] A detailed study of this third order non-linearity is beyond the scope of this work.

We now examine the physical processes that give rise to such intense SHG from a center-symmetric material. One could posit that the SHG is generated from an odd layered system with the last layer generating an intense SHG owing to loss of inversion symmetry. However this would result in a broadband response of the SHG and not be limited to the polariton resonance, as shown in Fig. 2b. Moreover this strong fundamental wavelength dependence has been noted in several TMDC flakes of various thickness when they are pumped on and off the polariton resonance. Another possibility is the enhancement of the residual surface SHG rate due to an enhanced density of photonic states owing to a cavity mode that is resonant with either the SH or the fundamental wavelength. To investigate this possibility we perform FDTD simulations for a bulk material with a fixed $\chi^{(2)}$ using a broadband fundamental source, where we replicate the configuration used in our experiment and obtain an enhancement of $\approx 16$ times. See Supplementary Note 5 and Supplementary Figure S4b for more details. A similar enhancement has been reported in systems where a TMDC flake is coupled to passive resonant structures [24-26]. This passive enhancement factor however fails to explain the drastic enhancement of at least three orders of magnitude when the system is excited at the polariton resonance. To fully explain the experimental results we use a model with layers of alternating $\chi^{(2)}[15]$. Any crystal with inversion symmetry should possess zero second order non-linearity because the macroscopic polarization, $p^{(2)}(E)=-p^{(2)}(-E)$ leads to vanishing of all even nonlinear susceptibility under the electric dipole approximation. This is indeed true for $2 \mathrm{H}$ stacking of even number of layers in 2D TMDCs in which the inversion symmetry is restored. This cancellation of SHG for an even number of layers can be envisioned macroscopically as shown in Fig. 4a, where two successive MLs have alternative signs of the induced second order polarization, i.e., $\chi^{(2)}$ in the first layer is equivalent to $-\chi^{(2)}$ in the next layer, leading to a cancelling of the SHG for even number of layers in the far field. However, recent reports have shown that despite the existence of geometrical inversion symmetry in some systems, e.g., bilayer WSe2, SHG can arise by breaking the inversion symmetry of the induced charges. If the field is asymmetric across different layers of the 2D TMDC, the nonlinear polarizations add coherently, generating SHG. 
This is depicted in Fig. 4b where the nonlinear polarizations represented by arrows, are in opposite directions, but not of the same magnitude [35] . Recent work has additionally shown that artificially stacking 2D layers with a controllable twist angle between the layers can lead to enhancing or suppressing SHG, described by a superposition relation shown in Fig. 4b but with the arrows now pointing along different directions [36]. For the given polariton system, if we consider a pump wavelength at the fundamental frequency $\omega$ impinging from the top, the electric field distribution is not symmetric. In this case the SHG from different layers does not cancel each other in the far field as shown in Fig. 4c thereby resulting in the SHG signal that is observed in the experiment. This effect is further enhanced by the higher photonic density of states at the polariton branches. While this model captures the qualitative behavior of the SHG, it overestimates the value of the SHG response seen previously in a related work [15]. We classically model the enhancement in the nonlinear process by solving coupled linear equations at the fundamental frequency $\omega$, and at the SHG frequency $2 \omega$. To describe the SHG response of the system we model the WSe 2 as a mutlilayer system with layer thickness of $0.5 \mathrm{~nm}$. The only non-vanishing susceptibility terms are,

$$
\chi_{y y y}^{(2)}=-\chi_{y x x}^{(2)}=\chi_{x x y}^{(2)}=\chi_{x y x}^{(2)}
$$

where $\mathrm{y}$ is the armchair direction of the crystal lattice. We assume in the simulation that the incident polarization is aligned with the armchair direction, giving rise only to SHG polarized in the armchair direction. We model the nonlinear polarization in each layer as,

$$
p_{l}^{(2)}=\epsilon_{0} \chi_{l, y y y}^{(2)} E_{l, y}^{(\omega)^{2}}
$$

where 1 is the layer number, and $\chi_{l}^{(2)}$ is positive for odd layers, and negative for even layer counted from a fixed reference, and $E_{l, y}^{(\omega)}$ is the field distribution in layer 1 at the fundamental frequency. We then use this polarization current as the source for the SH signal. We use this model to describe the SHG from a ML and the polariton system. Interestingly, using this simple model we can observe an enhancement of around four orders of magnitude between SHG from ML, and the SHG from bilayer, which perfectly matches our experimental results. The polariton system shows enhanced SHG at lower polariton resonance with SHG efficiency close to that of a ML. We attribute this large SHG in the cavity coupled system near resonance due to the combination of the field enhancement and its spatial profile, which leads to an asymmetric distribution of nonlinear polarization. At the same time in bulk $\mathrm{WSe}_{2}$ outside the lower polariton resonance we found an almost negligible SHG owing to near perfect cancellation of the SH field of individual layers in the far field as shown in Fig $4 \mathrm{~d}$.

The model used here does not invoke non-local effects and remain well within the dipole approximation. The analysis shows that in layered systems like TMDCs, SHG should be treated as a collective effect of its constituent non-centrosymmetric ML. Finally we comment on the advantages of having a dielectric spacer layer. In the structure described in the text above, the use of air as a spacer layer allowed us to access narrower polariton modes in addition to the advantages mentioned earlier. However similar SHG responses can also be found in structures with PMMA spacers and a metal mirror. The resonances obtained in those systems are broader and have a lower enhancement due to their lower quality factor. We could do away with the bottom mirror and form self-hybridized polaritons in bulk TMDCs on glass or silicon substrates as reported in several works [9, 27]. However in these systems we are limited to bulks of thickness ranging only about from about $60 \mathrm{~nm}$ to $100 \mathrm{~nm}$. This is shown via various transfer matrix calculation for different device geometries in Supplementary Note 6 and Supplementary Figure 5. In summary, here we have demonstrated a platform to relax crystal symmetry requirements for second order non-linear response 
in bulk TMDC crystals via formation of exciton polaritons. The fact that we achieve strong nonlinear responses in bulk TMDCs reduces challenges with fabrication and integration into passive photonic platforms. Although we focus solely on the SHG process in the current work other $\chi^{(2)}$ processes like sum/difference frequency generation and optical parametric amplification can be explored in these systems. In addition, these polariton systems inherently possess a $\chi^{(3)}$ response that can lead to saturation of absorption, tunable index of refraction and single photon non-linearity via polariton blockade, thereby forming a versatile platform for a plethora of applications in the field of non-linear photonics and quantum optics. Furthermore, the power dependent saturation of the SHG can be used to estimate polariton-polariton interaction strengths in systems where traditional techniques of measuring contrasts in resonant laser reflection as a function of power are not feasible.

\section{Methods}

A DBR with center wavelength of $740 \mathrm{~nm}$ consisting of 10.5 periods of alternating $\mathrm{TiO} 2$ and $\mathrm{SiO} 2$ with thicknesses of $88 \mathrm{~nm}$ and $128 \mathrm{~m}$, respectively, was sputtered on a glass substrate, with $\mathrm{SiO} 2$ being the top layer. A $7.5 \mu \mathrm{m}$ by $7.5 \mu \mathrm{m}$ square hole was written with electron beam lithography (Elionix ELS G100) on a spin-coated positive electron beam resist (495PMMA A6) of thickness $440 \mathrm{~nm}$. A bulk WSe 2 crystal of 140nm thickness and size of 60x70 $\mu \mathrm{m}^{2}$ was exfoliated with the scotch tape method and transferred on to the square hole via the polypropylene carbonate (PPC) transfer technique in order to create the free-standing $\mathrm{WSe}_{2} .1$ In order to remove residue left by the PPC process, the structure was then soaked in chloroform for 2 hours at room temperature. The thicknesses of both the air hole and the $\mathrm{WSe}_{2}$ were measured with atomic force microscopy (Bruker Dimension FastScan). White light dispersion data was collected by using a broadband halogen light source and imaging the back aperture of a 50x objective with numerical aperture of 0.8 (Olympus MPLFLN) onto a Princeton Instruments Monochromator with an EMCCD Camera (Pixis 1024B).

For the SHG measurements, a Titanium-Sapphire laser (Coherent Mira) was used to pump at the fundamental wavelength of $832 \mathrm{~nm}$, while the SHG signal was detected through the Princeton Instruments Monochromator. A 500 nm short pass filter was used in the collection side to filter out the excitation beam. Linear polarization measurements were recorded by using a linear polarizer (LP) in the excitation path and a collinear LP in the collection path. For helicity resolved SHG measurements, the excitation circular polarization was determined by using an LP followed by a quarter-wave plate (QWP) to determine the left and right circularly polarized states of the excitation beam. In the collection path, an analyzer of a QWP followed by an LP was used to resolve the chiral response of the system.

Coupled Mode Theory In this work, the nonlinear response of bulk $\mathrm{WSe}_{2}$ is underpinned by coupling it to a photonic cavity in form of a Fabry-Perot resonator with its two reflective surfaces are a bulk $\mathrm{WSe}_{2}$, and the DBR as shown in Fig. 1a. The system represents two eigenmodes, represented by their respective frequencies $\omega_{a}$ and $\omega_{b}$. Here, $\omega_{a}$ represents the exciton resonance of the $\mathrm{WSe}_{2}$, and $\omega_{b}$ represents the resonance frequency of the photonic cavity which can be actively tuned with changing its height h. For an external excitation, the photonic cavity mode is indirectly excited, and the bulk $\mathrm{WSe}_{2}$ mode is excited directly from an impinging wave as shown in Fig. 1a. These dynamics can be captured by employing the coupled mode theory (CMT) to describe the coupled oscillators system. The two oscillators are the material resonance $\omega_{a}$ and the photonic cavity mode $\omega_{b}$. The material resonance mode is coupled to the radiation incident from above with radiation rate $\gamma_{r}$, while the cavity mode is coupled 
indirectly to the incident radiation. The two modes are coupled with coupling rate $\kappa$ thus forming the half matter half-light particles when $\kappa>\gamma_{r}$. The CMT governing equations are:

$$
\begin{gathered}
\frac{d a}{d t}=\left(i \omega_{a}-\gamma_{a}-\gamma_{r}\right) a+i \kappa b+\sqrt{2 \gamma_{r}} s_{+} \\
s_{-}=-s_{+}+\sqrt{2 \gamma_{r}} a \\
\frac{d b}{d t}=\left(i \omega_{b}-\gamma_{b}\right) b+i \kappa a
\end{gathered}
$$

where $\gamma_{a}$ and $\gamma_{b}$ are the absorption rates of the 2D TMDC, and the photonic cavity respectively while $\gamma_{r}$ is the radiative coupling rate. For oblique incidence, the material resonance $\omega_{a}$ does not change, the cavity resonance changes as

$$
\omega_{b}^{2}=\omega_{b 0}^{2}+c^{2} k_{\perp}^{2} \rightarrow \omega_{b} \approx \omega_{b 0}+0.5 \sin ^{2} \theta
$$

with $\mathrm{c}$ is the light speed, and $k_{\perp}$ is the normal wavenumber inside the cavity. We can solve the differential equations under slowly varying approximation and give the reflection as,

$$
R=\left|\frac{-\left(i \Delta \omega_{a}+\gamma_{a}+\gamma_{r}\right)\left(i \Delta \omega_{b}+\gamma_{b}\right)-\kappa^{2}+2 \gamma_{r}\left(i \Delta \omega_{b}+\gamma_{b}\right)}{\left(i \Delta \omega_{a}+\gamma_{a}+\gamma_{r}\right)\left(i \Delta \omega_{b}+\gamma_{b}\right)+\kappa^{2}}\right|^{2}
$$

Here, $\omega_{b 0}=2.23 \times 10^{15} \mathrm{rad} / \mathrm{s}, \omega_{a}=2.55 \times 10^{15} \mathrm{rad} / \mathrm{s}, \gamma_{a}=0.015 \omega_{b}, \gamma_{b}=0.0011 \omega_{a}, \gamma_{r}=0.05 \omega_{a}, \kappa=0.004 \omega_{b}$, calculated to match the experimental results.

\section{Acknowledgments}

M.K, R.B, V.M conceived the idea, M.K and R.B performed the experiments, A.M and A.A performed theoretical and computational studies, V.M and A.A supervised the experiment. All authors contributed to writing the manuscript. We thank Professor Wang Yao from The University of Hong Kong for useful discussions. Authors acknowledge DARPA Nascent Light-Matter Interactions program, ARO MURI (W911NF-17-1-0312) and Simons Foundation for support. All the devices were fabricated partially at the Nanofabrication facilities at the ASRC ,CUNY.

\section{Conflict of interest}

Authors declare no conflict of interest.

\section{Data Availability}

Raw data and analysis codes used in this manuscript are available upon reasonable request. 


\section{References}

[1] Kin Fai Mak et al. "Atomically thin MoS2: A new direct-gap semiconductor". In: Physical Review Letters 105.13 (2010), pp. 2-5. ISSN: 00319007. DOI: 10.1103/PhysRevLett.105.136805. arXiv: 1004.0546.

[2] Hualing Zeng et al. "Optical signature of symmetry variations and spin-valley coupling in atomically thin tungsten dichalcogenides”. In: Scientific Reports 3 (2013), pp. 2-6. ISSN: 20452322. DOI: 10.1038/srep01608. arXiv: 1208.5864.

[3] Gang Wang et al. "Colloquium: Excitons in atomically thin transition metal dichalcogenides". In: Rev. Mod. Phys. 90 (2 Apr. 2018), p. 021001. DOI: 10.1103/RevModPhys.90.021001. URL: https://link.aps.org/doi/10.1103/RevModPhys . 90.021001.

[4] Di Xiao et al. "Coupled Spin and Valley Physics in Monolayers of $\mathrm{MoS}_{2}$ and Other Group-VI Dichalcogenides". In: Phys. Rev. Lett. 108 (19 May 2012), p. 196802. DOI: 10.1103/PhysRevLett.108.196802. URL: https://link.aps.org/doi/ 10.1103/PhysRevLett.108.196802.

[5] Xiaoze Liu et al. "Strong light-matter coupling in two-dimensional atomic crystals". In: Nature Photonics 9.1 (2014), pp. 30-34. ISSN: 17494893. DOI: 10.1038/nphoton.2014.304. arXiv: 1406.4826. URL: http://dx.doi.org/10.1038/ nphoton.2014.304.

[6] Zheng Sun et al. "Optical control of room-temperature valley polaritons". In: Nature Photonics 11.8 (Aug. 2017), pp. 491496. ISSN: 1749-4893. DOI: 10.1038/nphoton.2017.121. URL: https://doi.org/10.1038/nphoton.2017.121.

[7] Yen-Jung Chen et al. "Valley-polarized exciton-polaritons in a monolayer semiconductor". In: Nature Photonics 11.7 (July 2017), pp. 431-435. ISSN: 1749-4893. DOI: 10.1038/nphoton.2017.86. URL: https://doi.org/10.1038/nphoton. 2017. 86.

[8] S. Dufferwiel et al. "Valley-addressable polaritons in atomically thin semiconductors". In: Nature Photonics 11.8 (Aug. 2017), pp. 497-501. ISSN: 1749-4893. DOI: 10.1038/nphoton.2017.125. URL: https://doi.org/10.1038/nphoton.2017. 125.

[9] Battulga Munkhbat et al. "Self-Hybridized Exciton-Polaritons in Multilayers of Transition Metal Dichalcogenides for Efficient Light Absorption". In: ACS Photonics 6.1 (Jan. 2019), pp. 139-147. DOI: 10.1021/acsphotonics.8b01194. URL: https://doi.org/10.1021/acsphotonics.8b01194.

[10] Rahul Gogna, Long Zhang, and Hui Deng. "Self-Hybridized, Polarized Polaritons in ReS2 Crystals". In: ACS Photonics 7.12 (Dec. 2020), pp. 3328-3332. DOI: 10.1021/acsphotonics .0c01537. URL: https://doi.org/10.1021/acsphotonics . $0 c 01537$.

[11] Ruggero Verre et al. "Transition metal dichalcogenide nanodisks as high-index dielectric Mie nanoresonators". In: Nature Nanotechnology 14.7 (July 2019), pp. 679-683. ISSN: 1748-3395. DOI: 10 . 1038/s41565-019-0442-x. URL: https : //doi.org/10.1038/s41565-019-0442-x.

[12] Nardeep Kumar et al. "Second harmonic microscopy of monolayer MoS2". In: Physical Review B - Condensed Matter and Materials Physics 87.16 (2013), pp. 1-6. ISSN: 10980121. DOI: 10.1103/PhysRevB.87.161403. arXiv: 1302.3935. 
[13] Leandro M. Malard et al. "Observation of intense second harmonic generation from MoS2 atomic crystals". In: Physical Review B - Condensed Matter and Materials Physics 87.20 (2013), pp. 1-5. ISSN: 10980121. DOI: 10.1103/PhysRevB.87. 201401. arXiv: 1304.4289.

[14] G. A. Wagoner et al. "Second-harmonic generation in molybdenum disulfide". In: Journal of the Optical Society of America B 15.3 (1998), p. 1017. ISSN: 0740-3224. DOI: 10.1364/josab.15.001017.

[15] Yilei Li et al. "Probing Symmetry Properties of Few-Layer MoS2 and h-BN by Optical Second-Harmonic Generation". In: Nano Letters 13.7 (July 2013), pp. 3329-3333. ISSN: 1530-6984. DOI: 10.1021/nl401561r. URL: https://doi .org/10. $1021 / \mathrm{nl} 401561 \mathrm{r}$.

[16] Evgeny M. Alexeev et al. "Resonantly hybridized excitons in moiré superlattices in van der Waals heterostructures". In: Nature 567.7746 (Mar. 2019), pp. 81-86. ISSN: 1476-4687. DOI: 10.1038/s41586-019-0986-9. URL: https://doi.org/ $10.1038 / \mathrm{s} 41586-019-0986-9$.

[17] Kyle L. Seyler et al. "Signatures of moiré-trapped valley excitons in MoSe2/WSe2 heterobilayers". In: Nature 567.7746 (Mar. 2019), pp. 66-70. ISSN: 1476-4687. DOI: 10.1038/s41586-019-0957-1. URL: https://doi.org/10.1038/s41586019-0957-1.

[18] Kha Tran et al. "Evidence for moiré excitons in van der Waals heterostructures". In: Nature 567.7746 (Mar. 2019), pp. 7175. ISSN: 1476-4687. DOI: 10.1038/s41586-019-0975-z. URL: https://doi.org/10.1038/s41586-019-0975-z.

[19] Chenhao Jin et al. "Observation of moiré excitons in WSe2/WS2 heterostructure superlattices". In: Nature 567.7746 (Mar. 2019), pp. 76-80. ISSN: 1476-4687. DOI: 10.1038/s41586-019-0976-y. URL: https://doi.org/10.1038/s41586-0190976-y.

[20] Kaiyuan Yao et al. "Continuous Wave Sum Frequency Generation and Imaging of Monolayer and Heterobilayer TwoDimensional Semiconductors". In: ACS Nano 14.1 (2020), pp. 708-714. ISSN: 1936086X. DOI: 10.1021/acsnano.9b07555.

[21] Chiara Trovatello et al. "Optical parametric amplification by monolayer transition metal dichalcogenides". In: Nature Photonics 15.1 (Jan. 2021), pp. 6-10. ISSN: 1749-4893. DOI: 10.1038/s41566-020-00728-0. URL: https://doi.org/10. $1038 / \mathrm{s} 41566-020-00728-0$.

[22] Hatef Dinparasti Saleh et al. "Towards spontaneous parametric down conversion from monolayer MoS2". In: Scientific Reports 8.1 (Mar. 2018), p. 3862. ISSN: 2045-2322. DOI: 10.1038/s41598-018-22270-4. URL: https://doi .org/10. $1038 / \mathrm{s} 41598-018-22270-4$.

[23] Xinglin Wen, Zibo Gong, and Dehui Li. "Nonlinear optics of two-dimensional transition metal dichalcogenides". In: InfoMat 1.3 (2019), pp. 317-337. DOI: https://doi.org/10.1002/inf2.12024. eprint: https : //onlinelibrary . wiley . com/ doi/pdf/10.1002/inf2.12024. URL: https://onlinelibrary.wiley.com/doi/abs/10.1002/inf2.12024.

[24] Taylor K Fryett et al. "Silicon photonic crystal cavity enhanced second-harmonic generation from monolayer WSe 2". In: 2D Materials 4.1 (Dec. 2016), p. 015031. DOI: 10.1088/2053-1583/4/1/015031. URL: https://doi.org/10.1088/2053$1583 / 4 / 1 / 015031$. 
[25] Guangwei $\mathrm{Hu}$ et al. "Coherent steering of nonlinear chiral valley photons with a synthetic Au-WS2 metasurface". In: Nature Photonics 13.7 (July 2019), pp. 467-472. ISSN: 1749-4893. DOI: 10.1038/s41566-019-0399-1. URL: https : //doi.org/10.1038/s41566-019-0399-1.

[26] Haitao Chen et al. "Enhanced second-harmonic generation from two-dimensional MoSe2 on a silicon waveguide". In: Light: Science ES Applications 6.10 (Oct. 2017), e17060-e17060. ISSN: 2047-7538. DOI: 10.1038/1sa.2017.60. URL: https: //doi.org/10.1038/1sa.2017.60.

[27] Sebastian Busschaert et al. "Transition Metal Dichalcogenide Resonators for Second Harmonic Signal Enhancement". In: ACS Photonics 7.9 (Sept. 2020), pp. 2482-2488. DOI: 10.1021/acsphotonics .0c00751. URL: https://doi .org/10. 1021/acsphotonics.0c00751.

[28] Iacopo Carusotto and Cristiano Ciuti. "Quantum fluids of light". In: Reviews of Modern Physics 85.1 (2013), pp. 299-366. ISSN: 15390756. DOI: 10.1103/RevModPhys.85.299. arXiv: 1205.6500.

[29] Dawei Li et al. "Multimodal Nonlinear Optical Imaging of MoS2 and MoS2-Based van der Waals Heterostructures". In: ACS Nano 10.3 (Mar. 2016), pp. 3766-3775. ISSN: 1936-0851. DOI: 10.1021/acsnano.6b00371. URL: https://doi.org/ 10.1021/acsnano.6b00371.

[30] A. Verger, C. Ciuti, and I. Carusotto. "Polariton quantum blockade in a photonic dot". In: Phys. Rev. B 73 (19 May 2006), p. 193306. DOI: 10.1103/PhysRevB.73.193306. URL: https://link.aps.org/doi/10.1103/PhysRevB.73.193306.

[31] Li Bing Tan et al. "Interacting Polaron-Polaritons". In: Phys. Rev. X 10 (2 Apr. 2020), p. 021011. DoI: 10.1103/PhysRevX. 10.021011. URL: https://link.aps.org/doi/10.1103/PhysRevX.10.021011.

[32] Long Zhang et al. "Van der Waals heterostructure polaritons with moiré-induced nonlinearity". In: Nature 591.7848 (Mar. 2021), pp. 61-65. ISSN: 1476-4687. DOI: 10.1038/s41586-021-03228-5. URL: https://doi.org/10.1038/s41586-02103228-5.

[33] Fábio Barachati et al. "Interacting polariton fluids in a monolayer of tungsten disulfide". In: Nature Nanotechnology 13.10 (Oct. 2018), pp. 906-909. ISSN: 1748-3395. DOI: 10.1038/s41565-018-0219-7. uRL: https://doi.org/10.1038/s41565018-0219-7.

[34] Jie Gu et al. "Enhanced nonlinear interaction of polaritons via excitonic Rydberg states in monolayer WSe2". In: Nature Communications 12.1 (Apr. 2021), p. 2269. ISSN: 2041-1723. DOI: 10.1038/s41467-021-22537-x. URL: https://doi . org/10.1038/s41467-021-22537-x.

[35] Huakang Yu et al. "Charge-Induced Second-Harmonic Generation in Bilayer WSe2". In: Nano Letters 15.8 (Aug. 2015), pp. 5653-5657. ISSN: 1530-6984. DOI: 10.1021/acs.nanolett.5b02547. URL: https://doi.org/10.1021/acs. nanolett. $5 \mathrm{~b} 02547$.

[36] Wei-Ting Hsu et al. "Second Harmonic Generation from Artificially Stacked Transition Metal Dichalcogenide Twisted Bilayers". In: ACS Nano 8.3 (Mar. 2014), pp. 2951-2958. ISSN: 1936-0851. DOI: 10 . 1021 /nn500228r. URL: https : //doi.org/10.1021/nn500228r. 

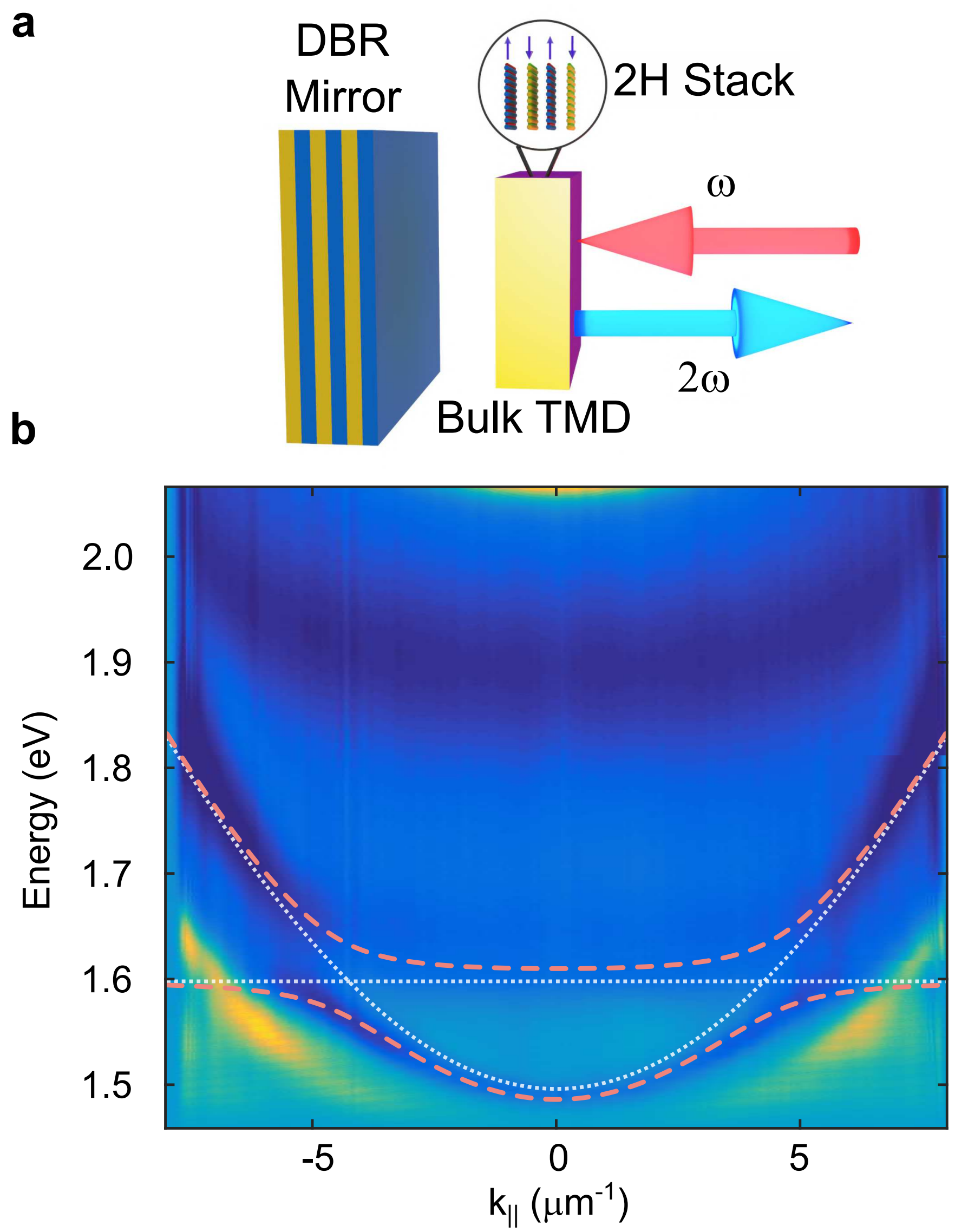

Figure 1: Fig 1a shows the schematic of the $\mathrm{WSe}_{2}$ polariton device. Fig $1 \mathrm{~b}$ shows the white light spectrum of the polariton system and a coupled oscillator model fit used to extract the polariton parameters are shown in pink dashed lines. 
a

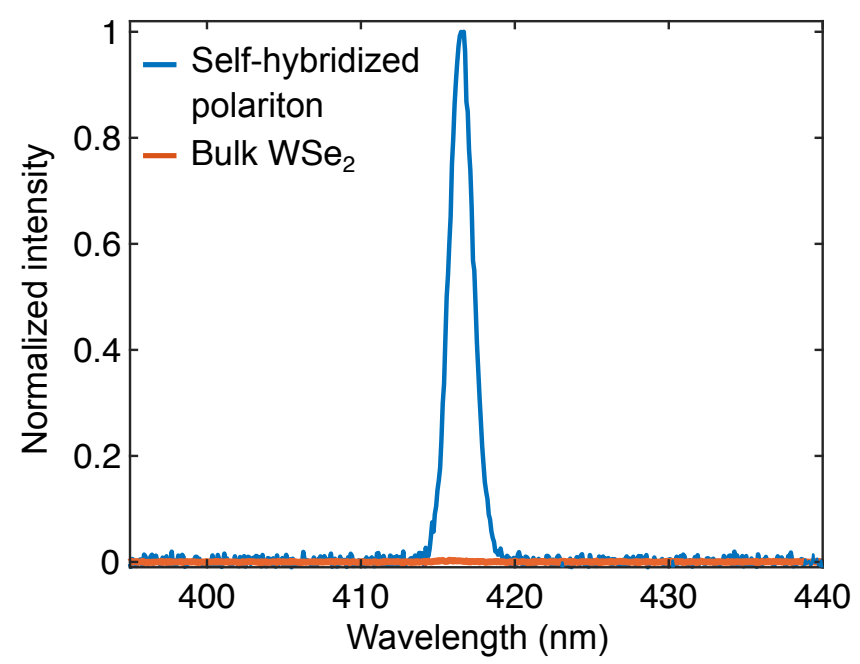

b

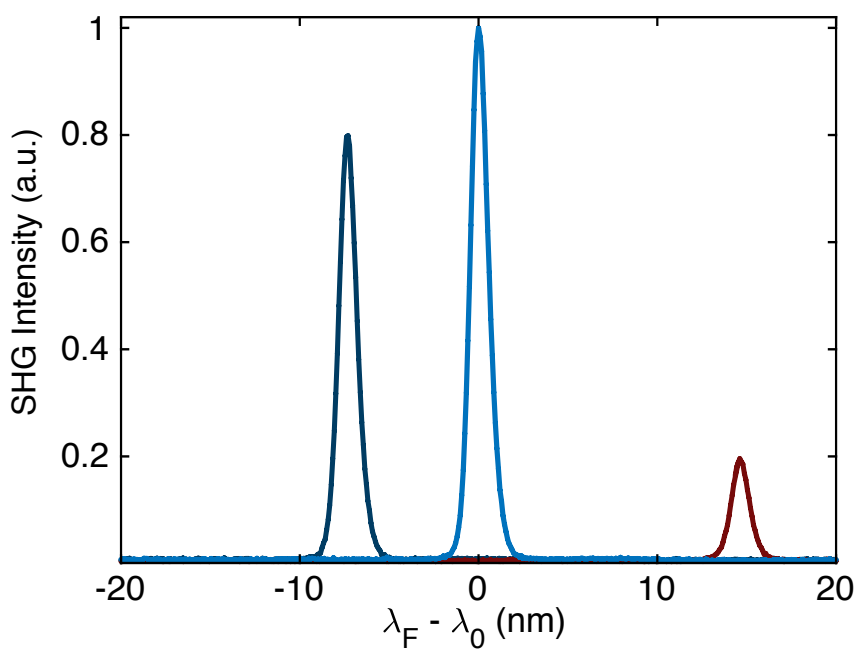

Figure 2: Fig 2a shows the SHG response of the polariton device with the fundamental resonant with wavelength at $\mathrm{k}_{\|}=0$. Fig $2 \mathrm{~b}$ compares the relative SHG intensity as a function of detuning of the fundamental from the wavelength at $\mathrm{k}_{\|}=0$.

a

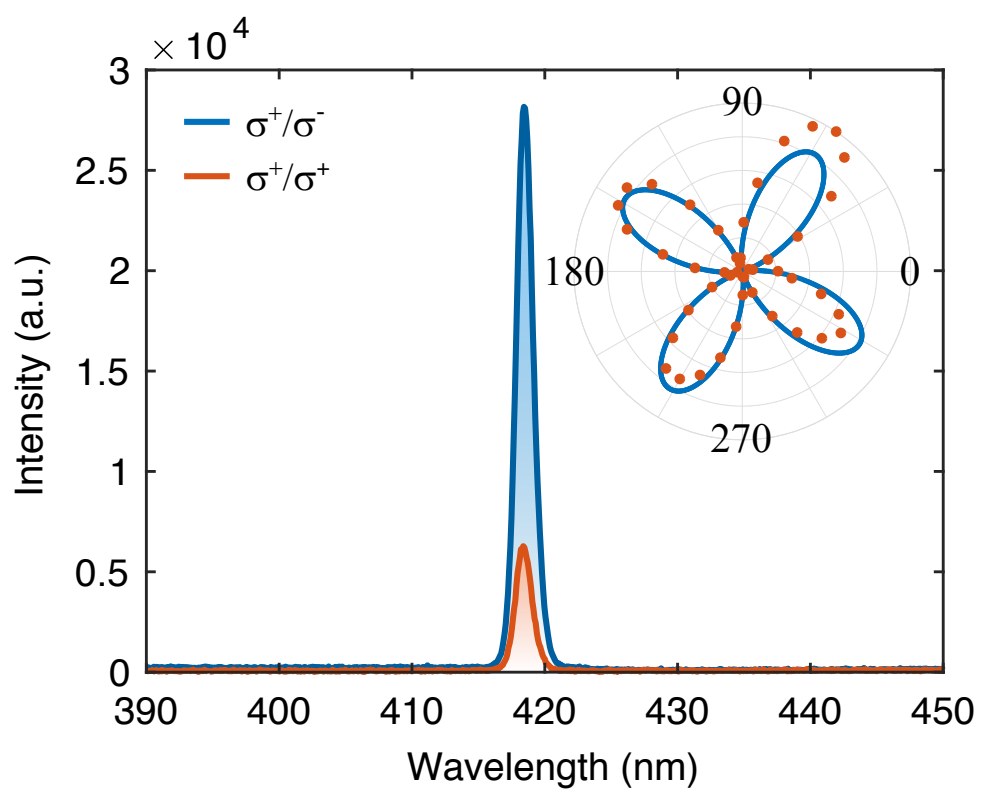

b

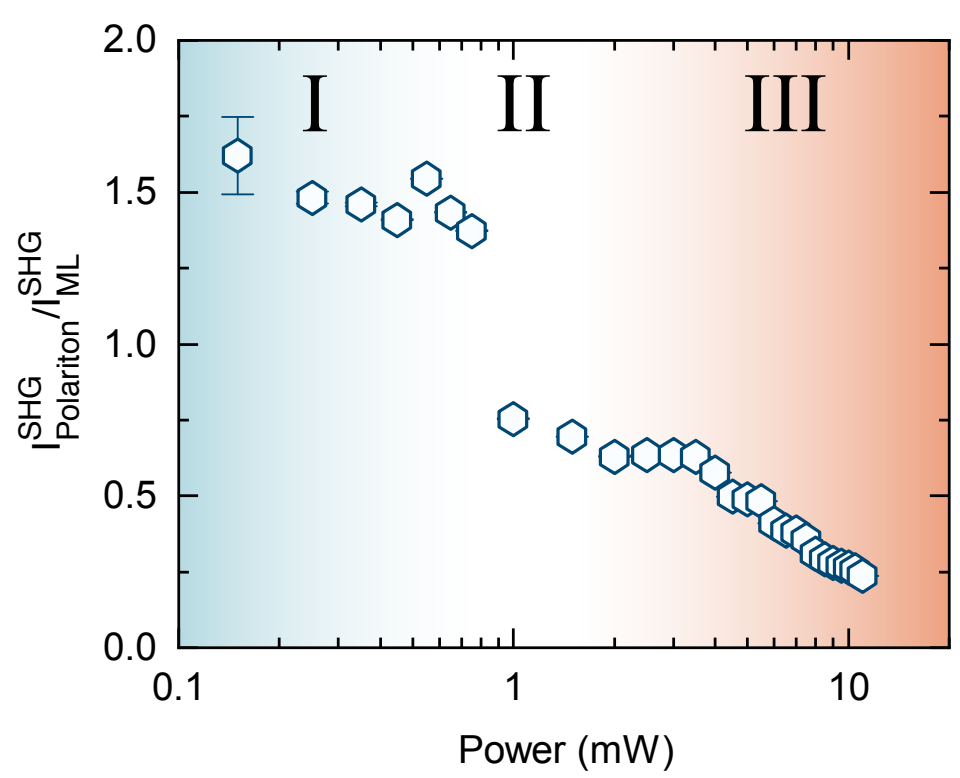

Figure 3: Fig 3a shows the SHG response as a function of input circular polarization . The helicity of the SHG is flipped as seen in ML systems. Inset shows the SHG response as a function of input linear polarization for a fixed analyzer.Fig $3 \mathrm{~b}$ shows the ratio of SHG intensity of a polariton system and a ML $\mathrm{WS}_{2}$ pumped at the same fundamental wavelength. Error bars represent fitting errors while extracting area under the curve of the SHG signals 
a

\section{Layer (1)}

Layer (2)

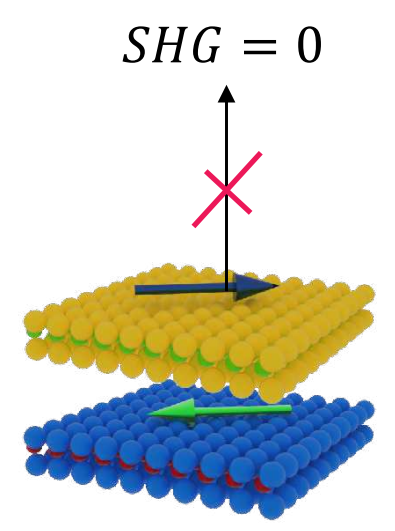

$p_{1}^{(2)}=-p_{2}^{(2)}$ b

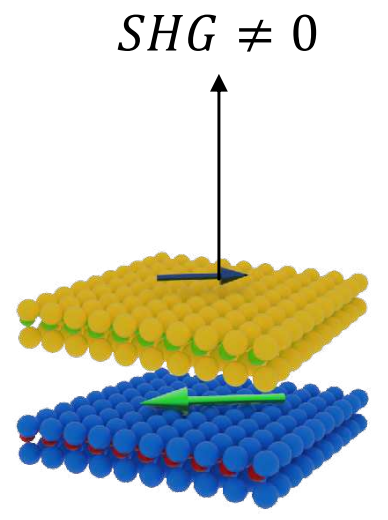

$p_{1}^{(2)} \neq-p_{2}^{(2)}$
C

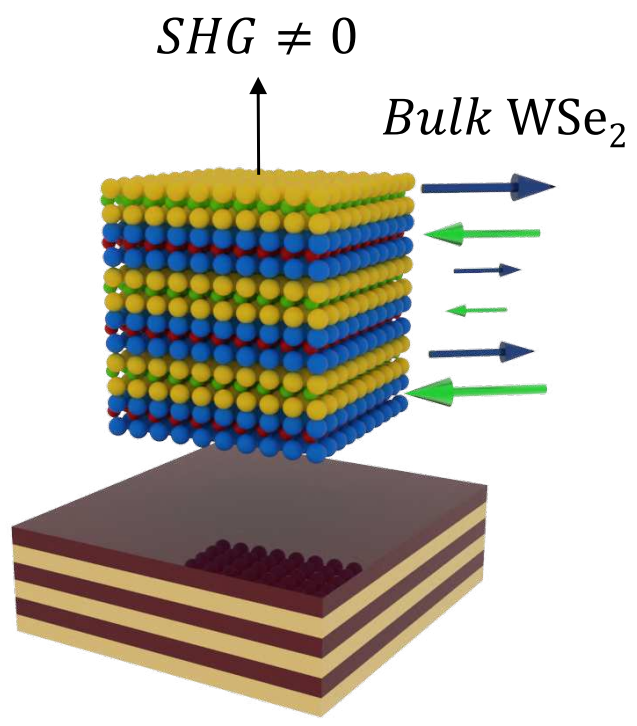

d Monolayer - Bulk WSe $_{2}$ on $\mathrm{SiO}_{2}$

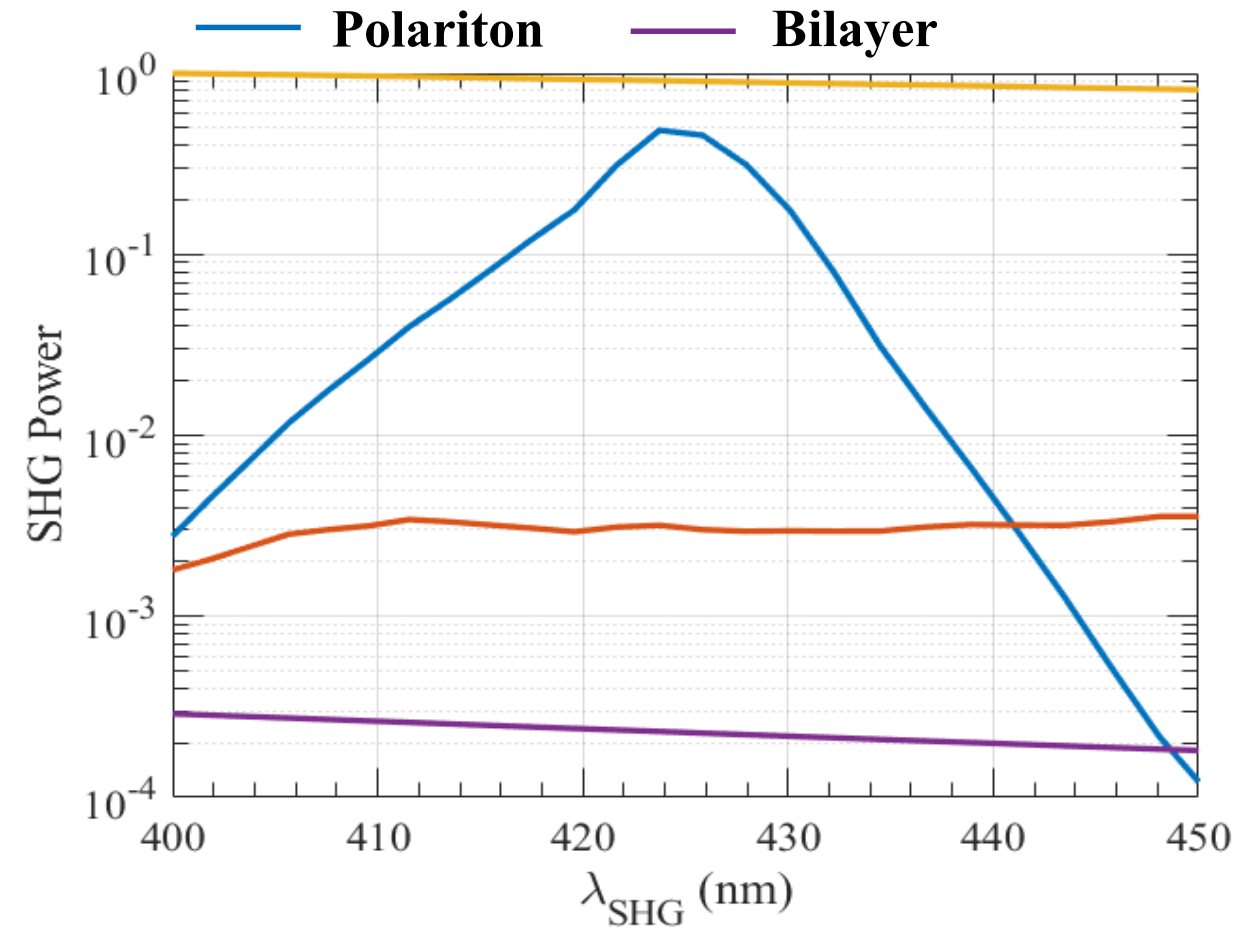

Figure 4: Fig 4a. shows $2 \mathrm{H}$ stacked bilayer $W S e_{2}$ with equal second order nonlinear polarization $\mathrm{p}$ and out of phase hence generating no SHG.Fig 4b. shows $2 \mathrm{H}$ stacked bilayer $W \mathrm{Se}_{2}$ with unequal and opposite p can generate nonzero SHG. (c) Enhancing the nonlinear polarization in bulk $W S e_{2}$ by coupling to a photonic cavity where the fundamental field significantly increase along with unequal second order polarization from individual layers in the bulk TMDC Fig 4d. shows normalized SHG power calculated for the structures in (c) with bulk TMDC height of $140 \mathrm{~nm}$. The ratios of the SHG at the SH wavelength 425 nm are,0.9 : $0.5: 0.003: 0.0002$ for the ML, polariton system, Bulk on $\mathrm{SiO}_{2}$, bilayer, respectively 


\section{Supplementary Files}

This is a list of supplementary files associated with this preprint. Click to download.

- supplementaryinfo050921.pdf 